\section{Personage Column: Prof. Ding Ma, M.D., Ph.D.}

Prof. Ding Ma was born in April 1957 in Kunming, China. He graduated from Tongji Medical College and gained M.D. degree in 1986 and Ph.D. degree in 1990. In 1992 Dr. Ding Ma went to Southwestern Medical Center of Texas University at Dallas to receive his postdoctoral training. He worked on the filed of tumor metastasis and cancer immunology. Dr. Ma distinguished himself in several areas and became an integral and vital member of his research team. He rapidly rose to the position of assistant professor in 1993 and associate professor in 1994. He published extensively in the area of cancer immunology and biology and was responsible for developing a strong research program on the immunotherapy and gene therapy of intraocular tumors. In December 1997, Dr. Ma was recruited back to China by the Ministry of Education as one of the overseas outstanding scholars to be a leading scientist of his filed. He was appointed professor and chairman of the Department of Obstetrics and Gynecology, director of Cancer Biology Center in Tongji Hospital.

During the past 10 years, Dr. Ding Ma tried his great efforts to build his department as a strong clinical and research bases. His department was selected to be one of the three National Key Subjects of the field in 2002. His cancer biology center upgraded to be the State Key Laboratory of Tumor Invasion and Metastasis in Hubei province in 2004. He continued his research in the molecular biology of tumor metastasis and gene therapy. Besides, he engaged himself in several related areas like research of multi-drug resistance and cervical cancer vaccine. In 2000 Dr. Ma gained the National Outstanding Youth Foundation to support his research on molecular mechanism of tumor metastasis. He was appointed the leading scientist in charge of one 973 program of cancer metastasis by the Ministry of Science and Technology in 2002. He also got or finished six National Nature Scientific Grants as well as one " 863 " program.

His consistent research was involved in three closelyrelated scientific issues: 1) Establishment of animal models of metastasis. A transgenic murine model of human uveal melanoma and an orthoptic liver carcinoma mice model were developed to investigate the pathological characteristics of secondary liver metastasis, which had a very important applicative value in liver cancer associated study. 2) Screening and verifying a series of effective

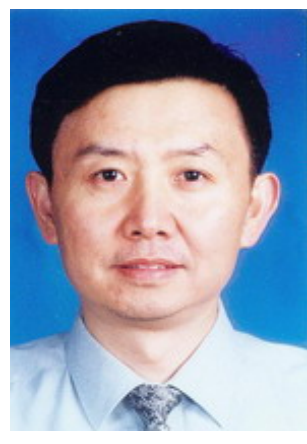

molecular suppressor genes that were closely associated with cancer metastasis through the systematic research in vivo and in vitro based on the established models, which were a stable bridge from the basic research to the applied research. 3) Establishment of effective vehicle systems targeted to liver carcinoma especially for metastatic cancer. A series of vehicles were developed including adenovirus, adenovirus associated virus and tumor-selective replication adenovirus vehicles in vitro and in vivo, which combined the cancer metastasis-targeting genes to block metastasis. A clinical trial of Adv-TK gene therapy on the liver transplantation of advanced liver cancer was performed to inhibit recurrence and metastasis and the primary results were promising.

Dr. Ding Ma devoted himself deeply in his clinical application, students training and research projects. He gained remarkable achievements especially in the research field of molecular regulation of tumor metastasis, targeting molecular therapy and tumor vaccine. He published more than 200 associated papers domestically and internationally, including 60 papers in the core journals overseas. Meanwhile, he published 6 academic books on tumor. He gained 7 national patents and 1 international patent. Due to his important contributions to his research field, Prof. Ma won the prestigious National Science and Technology Progress Award nominated by the Ministry of Education and the Natural Science Award of Hubei Province. Prof. Ma actively participated in student teaching. He was the editor-in-chief of the English academic teaching materials assigned by the Ministry of Health, vice chief editor of the national academic teaching materials for 8 year medical students. 